\title{
Sleep/Wake Classification using Cardiorespiratory Features Extracted from Photoplethysmogram
}

\author{
Parastoo Dehkordi ${ }^{1}$, Ainara Garde ${ }^{1}$, Guy A Dumont ${ }^{1,2}$, J Mark Ansermino ${ }^{2}$ \\ ${ }^{1}$ Department of Electrical and Computer Engineering, \\ ${ }^{2}$ Department of Anesthesiology, Pharmacology and Therapeutics, \\ The University of British Columbia, Vancouver, Canada
}

\begin{abstract}
Human sleep cyclically alternates between wakefulness and different sleep stages. There are various physiological changes that occur during wakefulness and sleep transitions. In particular, fluctuations occur in cardiorespiratory activity, mainly determined by the autonomic nervous system. The purpose of this study was to implement a multivariate logistic regression model to classify 30-second epochs of an overnight sleep dataset into awake and sleep states using the features extracted from the photoplethysmogram (PPG). The extracted features provided information about heart rate variability, respiratory activity, vascular tone and body movement.

Overnight PPG signals were collected using a smartphone-based pulse oximeter, simultaneously with standard polysomnography from 160 children at the British Columbia Children's hospital. The sleep technician scored all wake/sleep epochs throughout the PSG study. We divided the dataset into training data, used to develop the model using LASSO, and test data, used to validate the model.

The developed model was assessed epoch-by-epoch for each subject individually, and for the complete test dataset. The performance of the model on the full test dataset showed a median accuracy of $77 \%$, sensitivity of $80 \%$, and specificity of $70 \%$. Thus, providing a detailed epoch-byepoch analysis with at-home pulse oximetry alone is feasible with accuracy, sensitivity and specificity values above $70 \%$. However, the performance of the model might decrease when analyzing subjects with a high number epochs of wakefulness.
\end{abstract}

\section{Introduction}

Human sleep cyclically alternates between wakefulness and different sleep stages. Brain activity, eye movements and muscle tension change with progression through wakefulness and different sleep stages. Wake/sleep transitions also induce variations in heart rate, blood pressure, respiration and vascular tone, mainly regulated by the sympathetic and parasympathetic branches of the autonomic nervous system.

Polysomnography (PSG) is the gold standard for assessing sleep. In PSG, the recordings of brain activity (EEG), eye movement (EOG) and muscle activity (EMG) during sleep are used for sleep scoring. PSG requires that patients stay in the sleep laboratory with specialized equipment and an overnight attending sleep technician. The high cost and complicated procedure limits PSG tests to specialized sleep centers.

In recent years, the activity of the cardiorespiratory system has been monitored for the identification of wake/sleep transitions. Heart rate, heart rate variability (HRV) and respiration have recently been shown to be reliable tools for identifying sleep and wake in adults. Penzel et al. investigated the different linear and non-linear features of HRV in subjects with and without sleep apnea during various sleep stages [1]. Karlen et al. used spectral analysis of the electrocardiogram (ECG) and a respiratory signal recorded from a wearable sensor to classify sleep from wakefulness [2]. These studies demonstrated that sleep classification by monitoring the variation of heart and respiratory rate could attain results similar to sleep scoring achieved by the technicians using PSG recordings.

The purpose of this study is to differentiate the asleep from wakefulness state in overnight sleep, based on the activity of cardiorespiratory system using the pulse oximeter's photoplethysmogram (PPG). The PPG waveform reflects blood volume changes at the measuring site on the body (e.g., finger, forehead, and earlobe) and can be recorded using a pulse oximeter. A pulse oximeter uses the red and infrared absorption characteristics of oxygenated and deoxygenated hemoglobin. The PPG waveform contains information about a wide range of physiological parameters such as heart rate (HR), HRV, oxygen saturation $\left(\mathrm{SpO}_{2}\right)$, vascular tone, blood pressure, cardiac output and respiration. However, the majority of conventional pulse oximeters only provide information about $\mathrm{HR}$ and $\mathrm{SpO}_{2}$.

In this study, we extracted the relevant features 
associated with pulse rate variability (PRV), respiratory rate (RR), vascular tone and movement from the PPG signal to build a multivariate model with a minimum set of features to identify wakefulness from sleep. The PPG signals were recorded using the Phone Oximeter ${ }^{\mathrm{TM}}$, a mobile device that integrates a pulse oximeter with a smartphone [3]. The Phone Oximeter ${ }^{\mathrm{TM}}$ made the recording of PPG more convenient with the potential to be used at home or any other ambulatory environment use.

\section{Materials and methods}

\subsection{Database}

Following approval by the University of British Columbia Clinic Research Ethics Board (H11-01769) and informed parental consent, we recruited 160 children for this study. The children had been referred to the British Columbia Children's Hospital for overnight standard PSG. Children with a cardiac arrhythmia or abnormal hemoglobin were excluded from the study.

Standard PSG recordings included overnight measurements of ECG, electroencephalography (EEG), oxygen saturation $\left(\mathrm{SpO}_{2}\right), \mathrm{PPG}$, chest and abdominal movement, nasal and oral airflow, left and right electrooculography (EOG), electromyography (EMG) and video capture. The PSG recordings were performed with the Embla Sandman S4500 (Embla Systems, ON, Canada). The PSG was later manually scored by a sleep technician with sleep phases and respiratory events (e.g. apneas, hypopneas, and arousal) reported as the PSG log file.

In addition to PSG, the PPG was recorded simultaneously using the Phone Oximeter ${ }^{\mathrm{TM}}$ sampled at $62.5 \mathrm{~Hz}$ with 32-bit resolution.

\subsection{Preprocessing and sleep labelling}

All PPG signals recorded using the Phone Oximeter ${ }^{\mathrm{TM}}$ were divided into 30-second epochs after baseline removal and smoothing with a Savitzky-Golay FIR filter (order 3, frame size 11 samples). The pulse peaks of the PPG within each epoch were located using a peak detection algorithm based on zero-crossing. No attempt was made to distinguish normal pulses from others.

All epochs were scored as wakefulness and sleep based on the labels in the PSG event log file.

\subsection{Feature extraction}

For each 30-second epoch of the PPG signal, features associated with pulse rate variability (PRV), respiratory rate (RR), vascular tone and movement were extracted (Table I).

\section{$P R V$ features}

PRV reflects the variation of heart rate and is extracted from the pulse-to-pulse time intervals (PPIs) of PPG. In the time domain, three parameters were extracted from the PPIs time series, including the mean of the PPIs ( $m P P$ ), the standard deviation of the PPIs (SDPP) and the root mean square of the difference of the successive PPIs (RMSSD).

The PPIs time series were resampled into equivalent, uniformly spaced time series at a sampling rate of $4 \mathrm{~Hz}$ using the Berger algorithm. The power spectral density (PSD) was then estimated using a parametric autoregressive model with 1024 points and an order of 7 . The power in each of the following frequency bands was computed by determining the area under the PSD curve bounded by the band of interest: Very Low Frequency (VLF; 0.01-0.04 Hz), Low Frequency (LF; 0.04-0.15 Hz) and High Frequency (HF; 0.15-0.4 Hz). Normalized LF $(n L F)$ and normalized HF ( $n H F)$ powers were determined by dividing LF and HF powers by the total spectral power of PRV between 0.04 and $0.4 \mathrm{~Hz}$, respectively. The ratio of low-to-high frequency power ( $\mathrm{LF} / \mathrm{HF}$ ratio) was also computed.

\section{Vascular tone features}

Several morphological features were extracted from each pulse of the PPG signal within each epoch, to characterize vascular tone during different sleep stages.

-mAmp and stdAmp: the amplitude of each pulse was measured as the difference between the maximum of a pulse (peak) and the previous minimum (trough). mAmp and stdAmp were calculated as the average and standard deviation of the amplitude of all pulses within the epoch, respectively.

-mWidth half and stdWidth half: the width $_{\text {half }}$ of each pulse was calculated as the width at $50 \%$ of pulse height; later, $m$ Width half and stdWidth half were calculated as the average and standard deviation of width half of all pulses within the epoch, respectively.

-mWidth pulse $_{\text {and }}$ stdWidth $h_{\text {pulse }}$ : the width $_{\text {pulse }}$ of each pulse was calculated as the width at $10 \%$ of pulse height; later, mWidth $_{\text {pulse }}$ and stdWidth $h_{\text {pulse }}$ were calculated as the average and standard deviation of width $h_{\text {pulse }}$ of all pulses within the epoch, respectively.

-mTime rising $_{\text {and }}$ stdTime $_{\text {rising: }}$ the mean and standard deviation of Timerising (the time for a pulse takes to reach its peak) of all pulses within the epoch were computed.

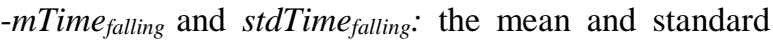
deviation of Time falling (the time for a pulse takes to reach its trough) of all pulses within the epoch were computed.

-mSlope and stdSlope: the mean and standard deviation of rising slope of all pulses within the epoch

$-p w v$ : For each epoch, pulse wave variability ( $p w v)$ was estimated as:

$$
p w v=\frac{\max (a m p)-\min (a m p)}{(\max (a m p)+\min (a m p)) / 2}
$$




\section{Respiratory features}

Respiratory activity may modulate the PPG in three different ways: 1) Respiratory-Induced Intensive Variation (RIIV): a baseline (DC) modulation caused by changes in venous return due to changes in intrathoracic pressure during the respiratory cycle, 2) Respiratory Induced Amplitude Variation (RIAV): an amplitude modulation caused by changes in left ventricular stroke volume, due to changes in intrathoracic pressure throughout the respiratory cycle, and 3) Respiratory Induced Frequency Variation (RIFV): a variation in heart rate that occurs during the respiratory cycle known as respiratory sinus arrhythmia. Since the respiratory rate changes during wakefulness and sleep states, in this study, RIAV, RIIV and RIFV were estimated from each 30-second epoch of PPG based on the method proposed by Karlen et al [4]. Then, the respiratory rates, $R R_{R I A V}, R R_{R I I V}$ and $R R_{R I F V}$, were estimated as the maximum value at the peak frequencies in respiratory bands of the power spectrum of RIAV, RIIV and RIFV (0.15-1 Hz), respectively.

\section{Movement features}

In actigraphy, accelerometers worn on the body are used to measure body acceleration and estimate the level of human activity. In sleep study applications the periods of low activity are considered as sleep. The PPG signal is usually readily corrupted by motion artifacts due to movement during data recording. In this study, in the absence of body acceleration measures, we used the motion artifacts in the PPG signal as the sign of body movement associated with restlessness.

- artifact $_{\text {epoch: }}$ the PPG signal was segmented into pulses and for each pulse a signal quality index (SQI) was calculated ranging from 0 to 100 (from low to high quality) based on cross-correlation of consecutive pulses [5]. Later, artifact $_{\text {epoch }}$ feature was assigned to each epoch according the following rules:

- artifact $_{\text {epoch }}=0$, if all pulses of the epoch have an SQI higher than 80 .

- artifact $_{\text {epoch }}=1$, if less than four pulses of the epoch have an SQI lower than 80 (less than four pulses of the epoch contaminated with artifact)

- artifact $_{\text {epoch }}=2$, if more than four pulses of the epoch have an SQI lower than 80 (more than four pulses of the epoch contaminated with artifact)

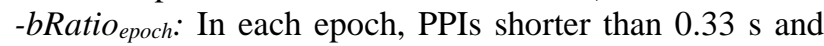
greater than $1.5 \mathrm{~s}$ were considered artifacts and labelled as abnormal intervals. BRatio $_{\text {epoch }}$ was estimated as the ratio of the number of normal intervals over the number of all intervals.

-skewepoch: a measure of the symmetry of each PPG epoch (or the lack of it) around the mean, defined as:

$$
\text { skew } w_{\text {epoch }}=\mu_{3} / \sigma^{3 / 2}
$$

where $\mu_{3}$ is the third central moment of the PPG epoch, $\sigma$ is the standard deviation of the PPG epoch.

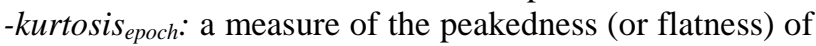
each PPG epoch distribution, relative to the normal distribution, defined by:

$$
\text { Kurtosis }_{\text {epoch }}=\mu_{4} /_{\sigma^{4}}-3
$$

Table 1. List of the features extracted from PPG within

\begin{tabular}{|c|c|}
\hline Feature & Description \\
\hline \multicolumn{2}{|r|}{ PRV } \\
\hline $\mathrm{mPP}$ & average of the PPIs \\
\hline SDPP* & standard deviation of the PPIs \\
\hline RMSSD* & root mean square of difference of successive PPIs \\
\hline Pow $_{\text {total }} *$ & total power of PRV \\
\hline VLF & power of PRV in VLF band $(0.01-0.04 \mathrm{~Hz})$ \\
\hline $\mathrm{nLF}$ & power of PRV in LF band (0.04-0.15 Hz) \\
\hline nHF & power of PRV in HF band $(0.15-1 \mathrm{~Hz})$ \\
\hline $\mathrm{LF} / \mathrm{HF}^{*}$ & nLF/nHF ratio \\
\hline \multicolumn{2}{|r|}{ Vascular tone } \\
\hline $\mathrm{mAmp}_{\text {pulse }} *$ & average of the pulse amplitudes in an epoch \\
\hline stdAmp pulse & standard deviation of the pulse amplitudes in an epoch \\
\hline mWidth $_{\text {half }} *$ & average of the width at $50 \%$ of pulse heights in an epoch \\
\hline stdWidth $_{\text {half }} *$ & $\begin{array}{l}\text { standard deviation of the width at } 50 \% \text { of height of } \\
\text { pulses of each epoch }\end{array}$ \\
\hline mWidth $_{\text {pulse }} *$ & average of the width at $10 \%$ of pulse heights in an epoch \\
\hline stdWidth & $\begin{array}{l}\text { standard deviation of the width at } 10 \% \text { of height of all } \\
\text { pulses within the epoch }\end{array}$ \\
\hline mTime $_{\text {rising }}$ & average of Time $_{\text {rising }}$ of all pulses in an epoch \\
\hline stdTime $_{\text {rising }} *$ & standard deviation of Time ${ }_{\text {rising }}$ all pulses in an epoch \\
\hline mTime $_{\text {falling }} *$ & average of Time $e_{\text {falling }}$ of all pulses in an epoch \\
\hline stdTime $_{\text {falling }} *$ & standard deviation of Time falling all pulses in an epoch \\
\hline mSlope & average of the rising slope of pulses within the epoch \\
\hline stdSlope* & $\begin{array}{l}\text { standard deviation of the rising slope of all pulses } \\
\text { within the epoch }\end{array}$ \\
\hline pwV* & pulse wave variability \\
\hline \multicolumn{2}{|r|}{ Respiratory Rates } \\
\hline $\mathrm{RR}_{\mathrm{RIAV}}$ & respiratory rate obtained from RIAV \\
\hline $\mathrm{RR}_{\mathrm{RIIV}} *$ & respiratory rate obtained from RIIV \\
\hline $\mathrm{RR}_{\mathrm{RIFV}} *$ & respiratory rate obtained from RIFV \\
\hline $\mathrm{RR}_{\text {mean }}$ & average of $\mathrm{RR}_{\mathrm{RIAV}}, \mathrm{RR}_{\mathrm{RIIV}}$ and $\mathrm{RR}_{\mathrm{RIFV}}$ \\
\hline$H F_{R I I V}$ & power in $0.15-1 \mathrm{~Hz}$ of the $R I I V$ power spectrum \\
\hline$H F_{R I A V}$ & power in $0.15-1 \mathrm{~Hz}$ of the RIAV power spectrum \\
\hline \multicolumn{2}{|r|}{ Movement } \\
\hline artifact $_{\text {epoch }}$ & $=0,1$ and 2 based on the SQI of each epoch \\
\hline bRatio $_{\text {epoch }} *$ & $\begin{array}{l}\text { ratio of the number of normal intervals over the number } \\
\text { of all intervals within each epoch }\end{array}$ \\
\hline skew $_{\text {epoch }} *$ & measure of the symmetry of each PPG epoch \\
\hline kurtosis $_{\text {epoch }}$ & measure of the peakedness of each PPG epoch \\
\hline
\end{tabular}
each epoch

\section{Multivariate model development and validation}

The subjects within the dataset were randomly divided into training (25\%) and test data (75\%). The features of epochs corresponding to subjects in the training dataset were used to train the wake/sleep classifier, to determine whether an epoch would be scored as sleep or wake. The least absolute shrinkage and selection operator (LASSO) method was used for training the classifiers to derive a final multivariate logistic regression model containing only the most important features. 
The validation of the model was then performed, epochby-epoch, for each subject within the test dataset. Individual classification results are represented through the accuracy, sensitivity, and specificity of epoch classification. The general performance of the model was then assessed using the distribution of the accuracy, sensitivity and specificity for the subjects in the testing dataset through the mean and 95\% confidence intervals (CI) of the quartiles (25, 50 [median] and 75 percentile).

Table 2. Classification results represented by the mean and $95 \%$ CI of the quartiles of the accuracy, sensitivity and specificity.

\begin{tabular}{lccc}
\hline $\begin{array}{c}\text { Classification } \\
\text { (testing set) }\end{array}$ & $\begin{array}{c}\text { Accuracy } \\
(\%)\end{array}$ & $\begin{array}{c}\text { Sensitivity } \\
(\%)\end{array}$ & $\begin{array}{c}\text { Specificity } \\
(\%)\end{array}$ \\
\hline 25 Percentile & $70[65,73]$ & $72[64,83]$ & $60[56,62]$ \\
50 Percentile & $\mathbf{7 8}[\mathbf{7 1 , 8 1 ]}$ & $\mathbf{8 0}[\mathbf{7 6 , 8 3}]$ & $\mathbf{7 0}[\mathbf{6 5}, \mathbf{7 3}]$ \\
75 Percentile & $83[79,84]$ & $88[83,93]$ & $76[69,83]$ \\
\hline
\end{tabular}

\section{Results and discussion}

The recordings of 14 subjects were discarded from the dataset due to inadequate length of sleep (less than 3 hours). After this exclusion, the total data set of 146 subjects was randomly divided into the training and test data sets with 100 and 46 subjects, respectively. The training dataset contained 84,291 epochs scored as sleep $(64,034)$ and wake $(20,257)$ based on the PSG event log file. Each epoch contains 31 features extracted from the PPG signal (Table 1). The LASSO wake/sleep model was trained and the 17 significant features (marked with * in Table 1) were selected based on the tuning parameter $\lambda=$ 9.408e-05.

For each subject in the test dataset, the significant features of each epoch were fed to the wake/sleep classifier to score the epoch as awake or sleep. The risk threshold was set at a probability of 0.725 .

The median accuracy, sensitivity and specificity for the subjects in the testing dataset were above 70\% (table 2); even for subjects at the lowest quartile of accuracy, sensitivity and specificity provided values above $60 \%$. Overall the model performed well at identifying wake/sleep epochs. However, some subjects showed unbalanced sensitivity-specificity values. These challenging cases corresponded to the risk threshold fixed at 0.725 especially for subjects with a very high or very low number epochs of wakefulness.

Our results, obtained with the Phone Oximeter ${ }^{\mathrm{TM}}$, are comparable with previous studies with more sophisticated approaches or devices for sleep staging based on monitoring the activity of cardiorespiratory system. In a recent study, Uçar et al. extracted 86 features from PPG recorded from 10 adult patients and used k-nearest neighbors classification and support vector machines to identify sleep from wakefulness [6]. The accuracy, sensitivity and specificity of trained model were reported as $73.36 \%, 0.81 \%$ and $0.77 \%$, respectively. However, the performance of the trained model was evaluated against $50 \%$ of epochs used in development of the model and has not been evaluated separately for each subject

\section{Conclusion}

This study shows that a multivariate logistic model based on features extracted from PPG, can be used classifying the 30-s epochs into wakefulness and sleep states. The model was created using LASSO with the significant PPG features trained using the training dataset. The validation results, obtained for each subject within the test dataset, provided acceptable results in identifying wake/sleep transitions. Thus, this model could enable an at-home pulse oximetry-based sleep screening with a more detailed epoch-by-epoch analysis, similar to the one provided by PSG.

\section{Acknowledgements}

The authors would like to thank the Pediatric Anesthesia Research Team and the clinical staff of the sleep laboratory at the BC Children's Hospital for their assistance conducting this study and also Aryannah Rollinson for her help in revising this manuscript.

\section{References}

[1] T. Penzel, J. W. Kantelhardt, L. Grote, J. H. Peter, and A. Bunde, "Comparison of detrended fluctuation analysis and spectral analysis for heart rate variability in sleep and sleep apnea,” IEEE Trans. Biomed. Eng., 5(10), p. 1143-51, 2003

[2] W. Karlen and D. Floreano, “Adaptive Sleep-Wake Discrimination for Wearable Devices,” IEEE Trans. Biomed. Eng., 58(4), p. 920-26, 2010

[3] W. Karlen, G. Dumont, C. Petersen, J. Gow, J. Lim, J. Sleiman, and M. Ansermino, "Human-centered phone oximeter interface design for the operating room," ICHI, 2011, p. 433-8

[4] W. Karlen, S. Raman, J. M. Ansermino, and G. Dumont, "Multi-parameter Respiratory Rate Estimation from the Photoplethysmogram," IEEE Trans. Biomed. Eng., 60, p. 1946-53, 2013

[5] W. Karlen, K. Kobayashi, J. M. Ansermino, and G. A. Dumont, "Photoplethysmogram signal quality estimation using repeated Gaussian filters and crosscorrelation,” Physiol. Meas., 33, p. 1617-29, 2012

[6] M. K. Uçar, M. R. Bozkurt, C. Bilgin, and K. Polat, "Automatic sleep staging in obstructive sleep apnea patients using photoplethysmography, heart rate variability signal and machine learning techniques," Neural Comput \& Applic, p. 1-16, 2016 